\title{
Research on Construction Method of Complex Electromagnetic Environment Based on Simulation Calculation
}

\author{
Zhenjie Zeng, Shiju Qi, Xiaofei Zhu, Xiaowei Shen, Bowen Jiang and Shangjun Cai \\ Rocket Force University of Engineering, Xi'an 710025, Chian
}

\begin{abstract}
The use of computer simulation technology to build complex electromagnetic environment is a hot topic in modern military science research. According to the characteristics of the radiation source in the real scene, the mathematical model of the radiation source is established. Then, according to the spatial relationship between the radiation source and the target position and the geographical features on the path, the wave propagation loss is calculated by the wave propagation algorithm. Synthesis of complex electromagnetic simulation signal; the most complex synthetic electromagnetic simulation signal with arbitrary waveform signal generator generated. The proposed method is simple and effective, and has the advantages of high flexibility, low cost and high reliability compared with the conventional complex electromagnetic environment construction method. It is suitable for constructing complex electromagnetic environment in large scale and large scene. Experiments verify the effectiveness of the generated complex electromagnetic signals.
\end{abstract}

Keywords-complex electromagnetic environment; construction method; signal generation; simulation calculation

\section{INTRODUCTION}

The impact of complex electromagnetic environment on weapons and equipment is manifold[1],Sometimes fatal, so it is urgent to assess the suitability of weapons and equipment in complex electromagnetic environments. One of the difficulties is how to use a low-cost method to build a complex electromagnetic environment that matches the actual battlefield conditions. Existing battlefield complex electromagnetic environment construction method is usually the use of communications, radar, navigation, optoelectronics and other signal generating and transmitting devices in a certain area or darkroom to generate the required electromagnetic environment[2-3],In order to achieve the true electromagnetic environment on the equipment for interference or environmental adaptability assessment. Taking into account the physical simulation, although the construction of open field can truly reflect the actual terrain shading, space dissemination of various factors, but also to engineering practice has increased the difficulty. Although the application of darkroom in semiphysical simulation can suppress the electromagnetic interference caused by internal electromagnetic multipath reflections and shield the external electromagnetic interference from relative silence[4-5],However, the darkroom is expensive, poor maneuverability, is not conducive to the assessment of the complex electromagnetic environment of the real battlefield[6].This paper provides a method for building a complex electromagnetic environment based on simulation calculation. By using computer simulation modeling, the electromagnetic sensitive model can replace the actual weapon equipment, and the equipment application plex electromagnetic environment formed by the radiation source And optimized. In this paper, the radiation source model and radio propagation model are firstly introduced from the compositional factors of the complex electromagnetic environment. Secondly, the calculation and signal synthesis method of the receiving electromagnetic power in the area of interest are analyzed in detail. Finally, the experiment and performance analysis of the complex electromagnetic environment signal generation are demonstrated. Applicability and effectiveness.

\section{A Single Radiation Source Forms THE ELECTROMAGNETIC ENVIRONMENT}

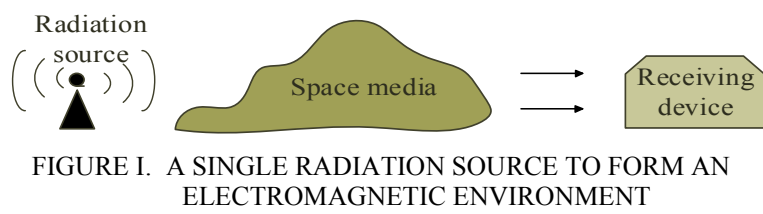

As shown in Figure 1, in order to accurately calculate and predict the field strength around the sensitive object and the received electromagnetic signals by using the known radiation source information, it is necessary to consider the space environment, the transmitter, the receiver, the antenna, the cable, etc. Series on the impact of radiation source signal. Therefore, we need to simulate the radiation source, radio wave propagation model, receiving equipment and so on. The specific electromagnetic calculation flow is shown in Figure 2.

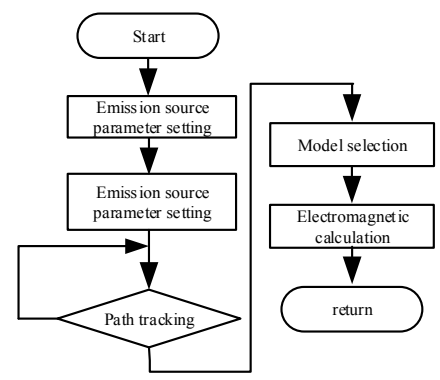

FIGURE II. ELECTROMAGNETIC CALCULATION FLOW CHART 


\section{A. Emitter Modeling}

Radiation source modeling refers to the generation of radiation signals based on the characteriste radiation source. In large-scale and large-scale scenarios, there are often multiple independent sources of radiation. The radiation source modeling is completed by generating the radiation source baseband signal according to the characteristic parameters of the radiation source. The main steps include:

\section{a:Radiation source characteristic parameters}

Suppose there are $\mathrm{N}$ radiation sources in the real scene, and each radiation source is independent. Each radiation source is characterized by parameters such as position, amplitude (or power), frequency, modulation, mounting height, gain, radiation pattern, polarization mode and so on. Radiation source radiation source characteristics can be described as:

$$
E_{i}=\{L L A, H, A, f, I Q, G, D F, P V\}_{i}
$$

Where Ei denotes the ith radiation source, LLA is the longitude, latitude and altitude of the location where the radiation source is located, $\mathrm{H}$ is the height of the antenna from the ground, $\mathrm{A}$ is the amplitude of the radiation source, $\mathrm{f}$ is the center frequency, IQ is the modulated baseband signal (The signal is determined by the modulation form), $G$ is the gain of the antenna, DF is the directivity function of the antenna and $\mathrm{PV}$ is the polarization direction vector of the antenna.

\section{b: Signals of radiation sources}

The radiation source signal is generated based on the characteristic parameters of the radiation source, which is:

$$
\begin{aligned}
S_{i}(n \Delta t, \theta, \Phi)= & D(\theta, \Phi) * P^{*} G^{*} \\
& {\left[I(n \Delta t)+j^{*} Q(n \Delta t)\right] }
\end{aligned}
$$

Among them $\Delta t$ is the sampling interval, $\theta$ is the pitch angle, $\Phi$ is the azimuth; $D(\theta, \Phi)$ is the direction of the graph function, $\mathrm{P}$ is expressed as normalized amplitude, $\mathrm{G}$ shows the gain of the antenna, $\left[I(n \Delta t)+j^{*} Q(n \Delta t)\right]$ represents the modulation signal, ${ }^{j=\sqrt{-1}}$. Sampling $\Delta t$ is based on the signal bandwidth, $\mathrm{N}$ is the number of radiation sources.

\section{B. Radio Wave Propagation Loss Calculation}

With the known target antenna position (LLAt) and installation height (Height), the path loss of each radiation source propagating to the target antenna is calculated in combination with the terrain on the path. The main steps include:

\section{a:Selection of radio wave propagation models}

Due to the spatiotemporal variation of the radiation source signal through the environmental medium, the complexity of the space wave propagation model and its characteristics changes. Therefore, the reasonable choice of space wave propagation model highlights is particularly important. Therefore, according to the radiation source frequency, the radiation source from the ground height, the target antenna distance from the ground height, select the appropriate propagation model.

\section{b: Propagation model parameter calculation}

According to the path between the radiation source and the target location (some require topographic parameters), calculate the parameters needed for the propagation model, such as the type of ground and the angle of cover.

c: Path loss calculation

$$
\begin{array}{r}
L_{i}=F\left(f_{i}, L L A_{i}, \text { Height }_{i}, L L A_{t},\right. \\
\text { Height } \left._{t}, \text { Terrain }\right)
\end{array}
$$

Which $F(\mathrm{x})$ represents the propagation model function expression, Terrain said the path parameter, $\mathrm{N}$ said the number of radiation sources.

\section{Received Power Calculation}

a, Receive antenna receiving area:

$$
A_{r}=\frac{G_{r} \lambda^{2}}{4 \pi}
$$

Convert to logarithm:

$$
G_{r}+36.55-20 \log 10 f
$$

$\mathrm{b}$, The received interference power is:

$$
J=S_{1}+A
$$

$$
\begin{aligned}
J= & P_{t}+30+G-71-20 \log _{10} d \\
& +\left(G_{r}+38.55-20 \log _{10} f\right)
\end{aligned}
$$

Finally can be expressed as:

$$
\begin{aligned}
J= & \left(P_{t}+30\right)+G+G_{r}- \\
& \left(32.45+20 \log _{10} d+20 \log _{10} f\right)
\end{aligned}
$$

$J$ is the interference power $(\mathrm{dBm})$ received by the receiver, $P t$ is the transmitter power $(\mathrm{dBw}), G$ is the transmit antenna gain $(\mathrm{dB}), G_{r}$ is the receive antenna gain, $d$ is the distance $(\mathrm{km})$ and $f$ is the operating frequency $(\mathrm{MHz}), A_{r}$ is the receiving area $\left(\mathrm{m}^{2}\right)$.

\section{COMPLEX ELECTROMAGNETIC ENVIRONMENT SimUlation SignAL SYNTHESIS CALCULATION}

The complex electromagnetic environment formed by multiple radiation sources is integrated and superposed at a certain position in space (ie, the spatial sampling points). The 
interpolation method is adopted to calculate the parameter characteristics. The mathematical simulation of the computer is the complex electromagnetic environment at that point. The observed value is compared with the theoretical value.

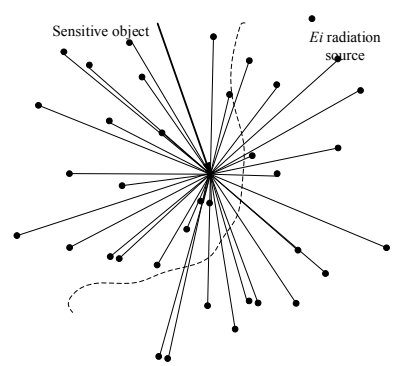

FIGURE III. COMPLEX ELECTROMAGNETIC ENVIRONMENT EQUIPMENT DISTRIBUTION

As shown in Figure 3, sensitive objects distributed in space are subject to electromagnetic interference from a variety of artificial and natural radiation sources of both our own and our own. Sensitive objects can act as both a radiation source and a sensitive body, and are maneuverable. Therefore, the electromagnetic environment of a sensitive object deployed in this location can be calculated at a point in an optional space complex electromagnetic environment[7].

\section{A. Complex Electromagnetic Simulation Signal Synthesis}

In the known target antenna receiving area $(A t)$, pattern ( $\left.D_{t}(\theta, \phi)\right)$ and polarization $(P i)$ cases, the synthesis of the received signal, the main steps include:

\section{a:Radiation source signal reception}

According to the radiation source position and the target position, calculate the visual axis; Determine the azimuth angles $\theta_{i \rightarrow t}$ and pitch angles $\phi_{i \rightarrow t}$ of the boresight at the radiation source; Determine the azimuth angle $\theta_{t \rightarrow i}$ and pitch angle $\phi_{t \rightarrow i}$ of the visual axis at the target[8]; Determine the polarization loss $L P_{i}$ of the radiation source antenna polarization and the target antenna polarization. Therefore, each radiation source signal received by the target antenna is:

$$
\begin{aligned}
R_{i}(n \Delta t, f)= & S_{i}\left(n \Delta t, \theta_{i \rightarrow t}, \phi_{i \rightarrow t}\right) * L_{i} * A_{t} \\
& * D_{t}\left(\theta_{t \rightarrow i}, \phi_{t \rightarrow i}\right) * L P_{i}
\end{aligned}
$$

Where $\mathrm{i}=1 \ldots \ldots \mathrm{N}, \mathrm{N}$ represents the number of radiation sources.

\section{$\mathrm{b}:$ Synthesis of radiation source signals}

According to the frequency of the radiation source $f_{i}=(\mathrm{i}=1 \ldots \mathrm{N})$, the synthesis frequency $R F$ and the synthesis signal sampling frequency are determined; each received signal is frequency transformed and resampled, get $R_{i}(n \Delta t)$. The resulting composite signal is thus expressed as:

$$
R(n \Delta t)=\sum_{i=1}^{N} R_{i}(n \Delta t)
$$

\section{B. Complex Electromagnetic Signal Generation}

According to the synthesized signal $R(n \Delta t)$ and the synthesized frequency $R F$, the digital electromagnetic susceptibility model of the equipment can be directly evaluated and evaluated, and a semi-physical simulation platform can also be set up with the electromagnetic signal transmitting equipment to generate the radio frequency signal with an arbitrary waveform signal generator.

\section{EXPERIMENT AND PERFORMANCE ANALYSIS}

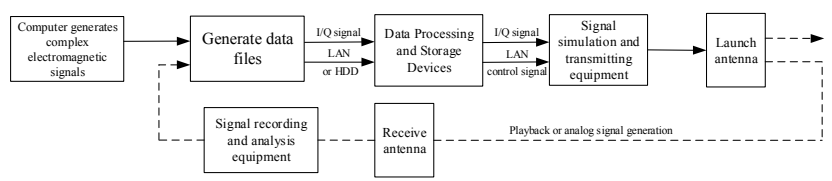

FIGURE IV. THE ACTUAL SIGNAL GENERATED BY THE OVERALL DESIGN PROCESS

In order to more directly verify the simulation results and the actual environment of the signal consistency, as shown in Figure 4 using the simulation-driven emission device to generate the actual signal method, the first computer simulation to generate electromagnetic baseband signals, I/Q through data processing and The data storage is loaded into the signal simulation and transmitting equipment, radio frequency signal is generated through the antenna, and the signals are processed and analyzed on the signal recording and analyzing equipment. In the process of data flow transmission, MATLAB is used to accurately solve the complex electromagnetic equations and plot the characteristics of the field state model[9]. The corresponding data model is established for the radiation source, and the generated signal is converted to $\mathrm{I} / \mathrm{Q}$ and stored separately, By reading the stored information in LabVIEW driver vector signal generator hardware unit, thus generating the actual signal.

\section{A. Experimental Design}

1.Single emitter signal simulation

LFM (Linear Frequency Modulated signal) As a typical non-stationary signal, due to its strong anti-interference ability and good characteristics of low interception rate, as a single radiation source signal simulation has better simulation results.

Test parameters:

(1)Sampling frequency $\left(F_{S}\right): 10 \mathrm{MHz}$

(2)Pulse Width $(P W): 20 \mu \mathrm{Hz}$

(3)Pulse repetition frequency $(P R F): 1000 \mathrm{~Hz}$

(4)FM signal bandwidth $(B W): 5 \mathrm{MHz}$

(5)Pulse cycle (T):5000

(6)Magnitude( $A): 1 \mathrm{~V}$ 


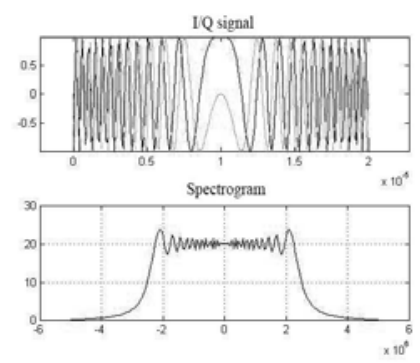

FIGURE V. LFM SIGNAL TIME-FREQUENCY ANALYSIS

\section{Mixed radiation sources}

The mixed signal is composed of radar echo signal, chirp signal and Rayleigh distributed clutter signal.

Test parameters:

(1)Baseband signal amplitude $(A): 5 \mathrm{~V}$

(2)Carrier frequency $\left(f_{0}\right): 10 \mathrm{MHZ}$

(3)Pulse Width $\left(P_{W}\right): 42 \mu \mathrm{s}$

(4)Sampling frequency $\left(F_{S}\right): 10 \mathrm{MHz}$

(5)Signal bandwidth $(B W): 2 \mathrm{MHz}$

(6)Pulse repetition period $(P R T): 240 \mu \mathrm{s}$

(7)Noise power:-12dB

(8)Shape parameters $(p): 1.5$

(9)Size parameters $(\sigma): 1$

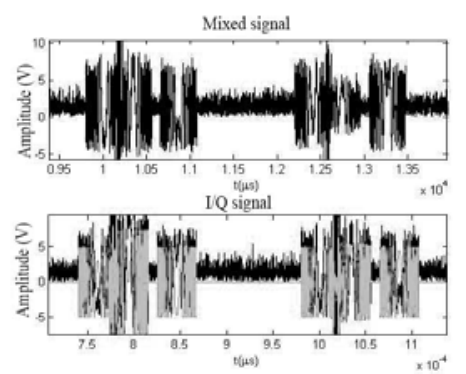

FIGURE VI. MIXED SIGNAL DISPLAY

\section{B. Other Conditions}

This experiment uses NI PXIe-5644R vector signal generator produced by the company. The VST features up to $80 \mathrm{MHz}$ of real-time bandwidth and 24-bit high-speed digital I / $\mathrm{O}$ with an adjustable center frequency of $65 \mathrm{MHz}$ to $6 \mathrm{GHz}$ for user-programmable development with LabVIEW FPGA.

The purpose of this experiment is to verify the feasibility of constructing a complex electromagnetic environment and to generate a simulated signal in a complex electromagnetic environment. Therefore, the experiment uses a direct cable connection between the transmitter and the oscilloscope, and does not consider the actual antenna during the experiment Of gain and path loss, in the process of computer simulation, in accordance with the free-space propagation model for electromagnetic calculations.
C. The Results Show That the Interface Design

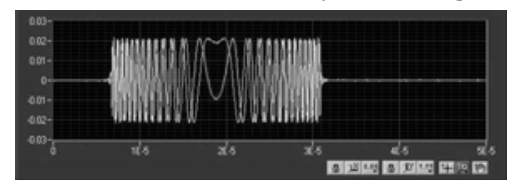

FIGURE VII. SHOWS THE ACTUAL RECEIVED LFM SIGNAL

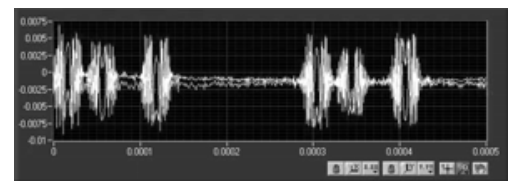

FIGURE VIII. SHOWS THE ACTUAL RECEIVED MIXED SIGNAL

Figure 7, Figure 8, respectively, for the LFM signal and mixed signal on the oscilloscope shows the results obtained. It can be seen that the single signal and the mixed signal can be clearly distinguished, which is in good agreement with the MATLAB simulation results in Figure 5 and Figure 6, which shows that the complex electromagnetic environment constructed by the simulation calculation is real and effective and has good scalability. Under the condition of hardware bandwidth, any complex electromagnetic signal can be simulated by computer and the reconstruction of complex electromagnetic environment can be realized.

\section{SUMMARY}

This study introduces a simulation based on complex electromagnetic environment simulation construction method, with practical significance. Firstly, the method can set parameters of any radiation source, which has the characteristics of strong operability, high simulation accuracy, controllable parameters and strong engineering practicability, and is more suitable for the complex electromagnetic environment in the battlefield under large-scale and large-scale scenarios Secondly, the comprehensive output of complex electromagnetic signals can provide the conditions and foundation for the demonstration and anti-interference performance evaluation of the equipment in the electromagnetic environment of complex battlefield to a greater extent.

\section{ACKNOWLEDGMENT}

Thanks to the guidance of my advisor, Professor Qi Shiju and my senior master Jiang Bowen, and thanks all teachers in the teaching and research office for their help, I was able to complete this paper. Thank you!

\section{REFERENCES}

[1] You Xiong.Foundation Environment Simulation Based on Virtual Reality Technology [J] .Journal of Surveying and Mapping, 2002,31 (2): 7-11.

[2] Ding Ling, Zhang Linxi.Study on Simulation of Complex Electromagnetic Environment in Battlefield [J]. Modern Electronics Technique, 2011,34 (14): 117-120 (in Chinese).

[3] Cui Ji-feng, Tian Yi-ming, Han Wei.Electronic Equipment Test Electromagnetic Environment Construction Method [J] .2012,27 (1): 6064. 
[4] Zhang Zhang, Song Haifeng, Zhang Zhili, Yue Yu.A comprehensive discussion on the performance of microwave anechoic chamber [J] Journal of Communications Security Conference, 2011: 16-20.

[5] Yang Weiwei. Research on the Flame Retardant and Microwave Absorbing Properties of Absorbing Materials in Darkroom [D]. Dalian: Dalian University of Technology, 2007: 5-9.

[6] SUN Guo-Zhi, LIU Shang-He, CHEN Jing-Ping, et al.Effects of Electromagnetic Environment Effects on Information War in Battlefield [J] .Military Operations and Systems Engineering, 2006,20 (3): 43-47.

[7] Li Xiuhe.Electromagnetic environment simulation of battlefield and training effect evaluation $[\mathrm{M}]$.Beijing: People's Liberation Army Publishing House, 2012: 207-209.

[8] Huang Boliang. DSP-based Marine Photoelectric Observer Stabilization Platform Control System Design [D]. Harbin: Harbin Engineering University, 2014.

[9] Liu Zebin, Zhou Zimei. Study on Prediction and Simulation of Electromagnetic Radiation Transmission Based on MATLAB [J] .Journal of Guangxi Radio and TV University, 2013,24 (4): 85-88. 